\title{
Concordancia de la filtración glomerular estimada según las fórmulas utilizadas en Colombia en pacientes con enfermedad renal crónica no en diálisis
}

\author{
Concordance of estimated glomerular filtration rate \\ according to the formulas used in Colombia for \\ patients with chronic kidney disease not on dialysis
}

\author{
Jorge Coronado-Daza, María Carolina Fragozo-Ramos, Lina Fernanda Reyes-Fontalvo \\ - Cartagena (Colombia)
}

DOI: https://doi.org/10.36104/amc.2020.1652

\section{Resumen}

Introducción: las guías colombianas de enfermedad renal crónica (ERC) recomiendan estimar la filtración glomerular (FG) con la fórmula de epidemiología de la enfermedad renal crónica (CKDEPI). No se han realizado estudios que comparen en la población colombiana la exactitud de esta fórmula con otras utilizadas en la práctica clínica.

Diseño y métodos: en 757 pacientes adultos evaluamos el desempeño para estimar la FG por las fórmulas de la modificación de la dieta en la enfermedad renal (MDRD-4), Cockroft-Gault (CG) y Cockroft-Gault corregida para la superficie corporal (CG-SC) comparada con la fórmula de CKDEPI. El desempeño se evaluó con mediciones del sesgo, precisión y exactitud.

Resultados: la media de la FG por CKD-EPI fue $37.32 \pm 12.71 \mathrm{~mL} / \mathrm{min} / 1.73 \mathrm{~m}^{2}$, por MDRD-4 de $39.8 \pm 13.2 \mathrm{~mL} / \mathrm{min} / 1.73 \mathrm{~m}^{2}$, por CG fue $35 \pm 12.6 \mathrm{~mL} / \mathrm{min}$ y por CG-SC de $34.52 \pm 11.34 \mathrm{~mL} /$ $\mathrm{min} / 1.73 \mathrm{~m}^{2}$. Todas las fórmulas tuvieron un sesgo con relación a la FG por CKD-EPI. La fórmula más exacta fue la FG estimada por MDRD-4 (FGeM) con una proporción de mediciones dentro del 15 y $30 \%$ en 97.1 y $99.74 \%$ de las mediciones respectivamente, y la menos exacta fue la FG estimada por $\mathrm{CG}(\mathrm{FGeCG})$ con 59.7 y $81.77 \%$ de las mediciones dentro del 15 y $30 \%$ respectivamente. El coeficiente de correlación de concordancia entre la FG por CKD-EPI y MDRD-4 fue de 0.97, con CG y CG-SC de 0.78 y 0.85 , respectivamente.

Conclusiones: la fórmula alterna más exacta para estimar la filtración glomerular en esta población colombiana es la MDRD-4 que tiene una alta concordancia con la fórmula de CKD-EPI. Se sugiere no utilizar la estimación de la FG con la fórmula de CG. (Acta Med Colomb 2020; 45. DOI: https://doi.org/10.36104/amc.2020.1652).

Palabras clave: tasa de filtración glomerular, ecuaciones de estimación, enfermedad renal crónica, función renal.

\footnotetext{
Abstract

Introduction: the Colombian guidelines for chronic kidney disease (CKD) recommend estimating glomerular filtration (GF) using the Chronic Kidney Disease Epidemiology Collaboration (CKD-EPI) equation. No studies have been performed in the Colombian population to compare the accuracy of this equation to that of others used in clinical practice.

Design and methods: we evaluated the GF estimation performance of the Modification of Diet in Renal Disease (MDRD-4), Cockroft-Gault (CG) and body surface area adjusted Cockroft-Gault (CG-BSA) equations against the CKD-EPI equation in 757 adult patients. Performance was evaluated using bias, precision and accuracy measurements.
}

Dr. Jorge Coronado-Daza: Internista-Nefrólogo. MSc, PhD. Profesor Asociado Departamento Médico, Grupos de Investigación "Alta Tensión" Universidad de Cartagena y "Nefrología ASOCOLNEF"; Dra. María Carolina FragozoRamos: Médico Internista; Dra. Lina Fernanda Reyes-Fontalvo: Médico Interno. Grupo de Investigación “Alta Tensión” Universidad de Cartagena. Cartagena (Colombia).

Correspondencia: Dr. Jorge Coronado-Daza, Cartagena (Colombia).

E-mail: jocodada@yahoo.es

Recibido: 07/II/2020 Aceptado: 12/VIII/2020 
Results: the mean GF by CKD-EPI was $37.32 \pm 12.71 \mathrm{~mL} / \mathrm{min} / 1.73 \mathrm{~m}^{2}$; by MDRD-4 it was $39.8 \pm 13.2 \mathrm{~mL} / \mathrm{min} / 1.73 \mathrm{~m}^{2}$, by CG it was $35 \pm 12.6 \mathrm{~mL} / \mathrm{min}$ and by CG-BSA it was $34.52 \pm 11.34 \mathrm{~mL} /$ $\mathrm{min} / 1.73 \mathrm{~m}^{2}$. All the equations had bias with respect to GF by CKD-EPI. The most accurate equation was GF estimated by MDRD-4 (MeGF) with 97.1 and $99.74 \%$ of measurements within 15 and $30 \%$, respectively; and the least accurate was GF estimated by CG (CGeGF) with 59.7 and $81.77 \%$ of the measurements within 15 and 30\%, respectively. The concordance correlation coefficient between GF by CKD-EPI and MDRD-4 was 0.97, with CG and CG-BSA at 0.78 and 0.85 , respectively.

Conclusions: the most accurate alternative equation for estimating glomerular filtration in this Colombian population is MDRD-4, which has a high concordance with the CKD-EPI equation. Estimation of GF with the CG equation is not recommended. (Acta Med Colomb 2020; 45. DOI: https://doi.org/10.36104/amc.2020.1652).

Key Words: glomerular filtration rate, estimation equations, chronic kidney disease, renal function .

\section{Introducción}

La estimación de la filtración glomerular (FG) es una importante herramienta para el clínico, utilizada rutinariamente para identificar la enfermedad renal crónica (ERC), monitorizar cambios en la función renal y ajustar la terapia farmacológica en algunos pacientes (1-5). Existen métodos más exactos que revelan los valores reales del filtrado glomerular como la depuración de inulina y de diversos radioisótopos $(6,7)$. Dada la complejidad y costo de estas técnicas, han sido en la práctica clínica sustituidas por las ecuaciones de estimación de la tasa de FG (TFG).

En la actualidad ha sido necesario determinar cuál de las ecuaciones de estimación de la TFG tiene una mayor precisión para clasificar al enfermo renal. A nivel mundial se ha establecido la concordancia entre las fórmulas de estimación de la filtración glomerular de Cockroft-Gault (CG), modificación de la dieta en la enfermedad renal de cuatro variables (MDRD-4) y la fórmula de epidemiología de la enfermedad renal crónica (CKD-EPI), al compararlas con la medición directa basada en iotalamato (8). Desde que la fórmula de CKD-EPI fue publicada, el desempeño de las ecuaciones se ha visto comparado en diferentes tipos de poblaciones identificando que la fórmula CKD-EPI es la más precisa (9-11) y por esto en las guías KDIGO 2012, se toma dicha fórmula como base para la clasificación de los pacientes con ERC (12).

Las guías colombianas de ERC 2016 (13), una adopción de las guías KDIGO, han tomado la CKD-EPI como la fórmula para estimar la filtración glomerular. Sin embargo, desde 2008 cuando se estableció la Cuenta de Alto Costo (CAC) en Colombia, la ecuación utilizada hasta la actualidad para definir los pacientes con ERC es la de CG-SC (14). El motivo de utilizar la fórmula de CG es por su facilidad de ejecutarla manualmente y no requerir disponibilidad de internet, lo cual es necesario para utilizar la fórmula MDRD-4 o la CKD-EPI.

El sentir de los clínicos es que, al seguir los lineamientos de la CAC, se están clasificando erróneamente pacientes con ERC, dado que se utiliza la fórmula de CG-SC para todos los pacientes sin tener en cuenta que esta no puede utilizarse en pacientes con edades extremas, pesos extremos, enfermedad muscular, amputados y/o vegetarianos $(15,16)$. El objetivo de nuestro estudio es establecer la concordancia de la medición de la TFG según la formula CG, CG-SC, MDRD-4 y CKDEPI en pacientes con ERC desde el estadio 3a (G3a) hasta el estadio 5 no en diálisis (G5ND).

\section{Población de estudio}

\section{Material y métodos}

Se realizó un estudio observacional en pacientes que acudieron a la consulta de nefrología en los años 2016 y 2017 de una institución prestadora de salud en la ciudad de Cartagena, Colombia.

Los criterios de inclusión fueron: 1) Pacientes adultos mayores de 18 años en seguimiento por ERC G3a - G5ND, 2) Pacientes con cálculo de la filtración glomerular por las fórmulas $\mathrm{CG}(\mathrm{FGeCG})$, la fórmula de $\mathrm{CG}$ corregida para la superficie corporal (FGeCG-SC), MDRD-4 y CKD-EPI.

Se excluyeron: embarazadas, amputados de miembros inferiores, vegetarianos, neoplasias activas, enfermedades musculares primarias o secundarias. Se recolectaron datos de: régimen de afiliación en salud, edad, sexo, peso, talla, índice de masa corporal (IMC), causa de la ERC y albuminuria y/o proteinuria.

\section{Estimación de la TFG}

Se realizó el cálculo de la TFG en todos los casos utilizando las ecuaciones de CG ( $\mathrm{mL} / \mathrm{min})$, CG-SC, MDRD-4 y CKD-EPI $\left(\mathrm{mL} / \mathrm{min} / 1.73 \mathrm{~m}^{2}\right)$ como se describe a continuación:

- FG estimada por Cockcroft-Gault (FGeCG) (15):

- Depuración de creatinina en hombres $=(140-$ edad $)$ $\times$ (peso) / (creatinina sérica $\times 72$ ).

- Depuración de creatinina en mujeres $=(140-$ edad $)$ $\times($ peso $) \times 0.85 /($ creatinina sérica $\times 72)$.

- FG estimada por Cockcroft-Gault corregida por superficie corporal (FGeCG-SC) (15):

- Hombres $=(140-$ edad $) \times($ peso $) \times\left(1.73 \mathrm{~m}^{2} /\right.$ superficie corporal $) /($ creatinina sérica $\times 72)$.

- Mujeres $=(140-$ edad $) \times($ peso $) \times\left(1.73 \mathrm{~m}^{2} /\right.$ superficie corporal) $x 0.85$ / (creatinina sérica $\times 72$ )

El cálculo de la superficie corporal se realizó con la fór- 
mula de Dubois (17): superficie corporal $\left(\mathrm{m}^{2}\right)=0.20247$ $\times$ altura $(1)^{0.725} \times$ peso $(\mathrm{kg})^{0.425}$

- FG estimada por MDRD de cuatro variables (FGeM) (18):

- 175 x (creatinina sérica / 0.95)-1.154 x (edad) -0.2030 x (0.742 si es mujer) x (1.210 si es de raza negra).

- $\quad$ FG estimada por CKD-EPI (FGeCKD-EPI) (19):

- $141 \times \min \{\text { creatinina } / k, 1\}^{\alpha} \times \operatorname{máx}$ \{creatinina / $k$, $1\}-1.209 \times 0.993$ edad [años] $\times 1.018$ [si es mujer] donde, $k$ es 0.7 para mujeres y 0.9 para hombres, $\alpha$ es -0.329 para mujeres y -0.411 para hombres.

Las unidades utilizadas en las ecuaciones fueron edad en años, peso en kilogramos $(\mathrm{kg})$, concentración sérica de creatinina $(\mathrm{Cr})$ en $\mathrm{mg} / \mathrm{dL}$ y superficie corporal $(\mathrm{SC})$ en $\mathrm{m}^{2}$.

\section{Categorías según la TFG}

La FG reducida, definida como TFGe $<60 \mathrm{~mL} / \mathrm{min} / 1.73$ $\mathrm{m}^{2}$ se clasificó en cuatro grupos según directriz de la guía KDIGO 2012 (12): 45-59 mL/min/1.73 m² (G3a), 30-44 $\mathrm{mL} / \mathrm{min} / 1.73 \mathrm{~m}^{2}$ (G3b), $15-29 \mathrm{~mL} / \mathrm{min} / 1.73 \mathrm{~m}^{2}$ (G4) y $<15$ $\mathrm{mL} / \mathrm{min} / 1.73 \mathrm{~m}^{2}$ (G5). Se consideró un estadio G5ND a los pacientes en categoría G5 en manejo conservador sin requerimiento de diálisis. Se describió la prevalencia para cada categoría según las ecuaciones en estudio.

\section{Análisis estadístico}

Aplicamos estadística descriptiva para las variables categóricas y cuantitativas, expresándolas como frecuencias, porcentajes, media y mediana de acuerdo con la naturaleza de cada variable.

Para el total de los pacientes nosotros calculamos el coeficiente de correlación de concordancia (CCC) (20) entre la TFG estimada por CKD-EPI y la TFG estimada por otras fórmulas MDRD-4, FGeCG y FGeCG-SC. De acuerdo con el CCC la concordancia se catalogó así: 1=perfecta; $>0.99=$ casi perfecta; 0.95 a $0.99=$ sustancial; 0.900 a $0.949=$ moderada; $y<0.900=$ pobre.

La FG estimada por la fórmula CKD-EPI fue considerada el estándar de oro (FGeSO) para este estudio. La FGeSO fue comparada mediante el análisis de Bland-Altman (21) (representación gráfica de la recta de regresión resultante de utilizar como variable dependiente la diferencia de valores de las medias de las fórmulas en comparación y como variable independiente el promedio de sus medias) con la FGeM, FGeCG y FGeCG-SC. Los límites del intervalo de dos desviaciones estándar alrededor de la media de las diferencias se consideraron como los límites de concordancia entre ambas mediciones, ya que incluye $95 \%$ de las diferencias observadas. Se estableció un límite de concordancia máximo de \pm 5 entre las fórmulas de estimación con relación a la FEeSO (CKD-EPI), teniendo en cuenta la definición de progresión acelerada de la ERC que establece es el descenso sostenido de la FG mayor de $5 \mathrm{~mL} / \mathrm{min} /$ año.

Se evaluó sesgo, precisión y exactitud entre las fórmulas comparadas. El sesgo fue evaluado como la media de las diferencias de las medias entre la FGeSO y la FGeM, la
FGeCG y la FGeCG-SC. La precisión se definió como el rango de los límites de concordancia de la media de las diferencias de las medias (sesgo) entre la FGeSO y las estimadas por la FGeM, la FGeCG y la FGeCG-SC. La exactitud se midió como el porcentaje de resultados estimados con las diferentes fórmulas dentro del 15\% (P15) y $30 \%$ (P30) de las mediciones con la FGeSO. Un P30 >90\% califica una fórmula como satisfactoria para la interpretación clínica (22). La mejor fórmula se definió como aquella con menor sesgo, menor rango de límites de concordancia (o mejor precisión) y mayor p30. Para el análisis se utilizaron los softwares estadísticos Stata 14 y XLSTAT Premium.

\section{Aspectos éticos}

Los principios éticos estuvieron en concordancia con la Resolución 8430 de 1993 de Colombia. Por ser un estudio de no intervención, con riesgo mínimo, no se solicitó consentimiento informado por escrito, sólo verbal; el estudio fue aprobado por la institución prestadora de salud. Se respetó la confidencialidad, integridad y transparencia de los datos.

\section{Resultados}

Se recolectaron datos de 855 pacientes de los cuales 757 fueron elegibles para el análisis final al cumplir los criterios de inclusión (Figura 1).

De los sujetos analizados $61.96 \%$ fueron hombres, $38 \%$ mujeres, pertenecientes al régimen contributivo $50.53 \%$, y $48.68 \%$ al subsidiado. La hipertensión arterial fue la principal causa de ERC (61.43\%), seguido por la diabetes mellitus (31.04\%); las glomerulopatías primarias y otras causas correspondieron al $7.53 \%$ restante. Las principales

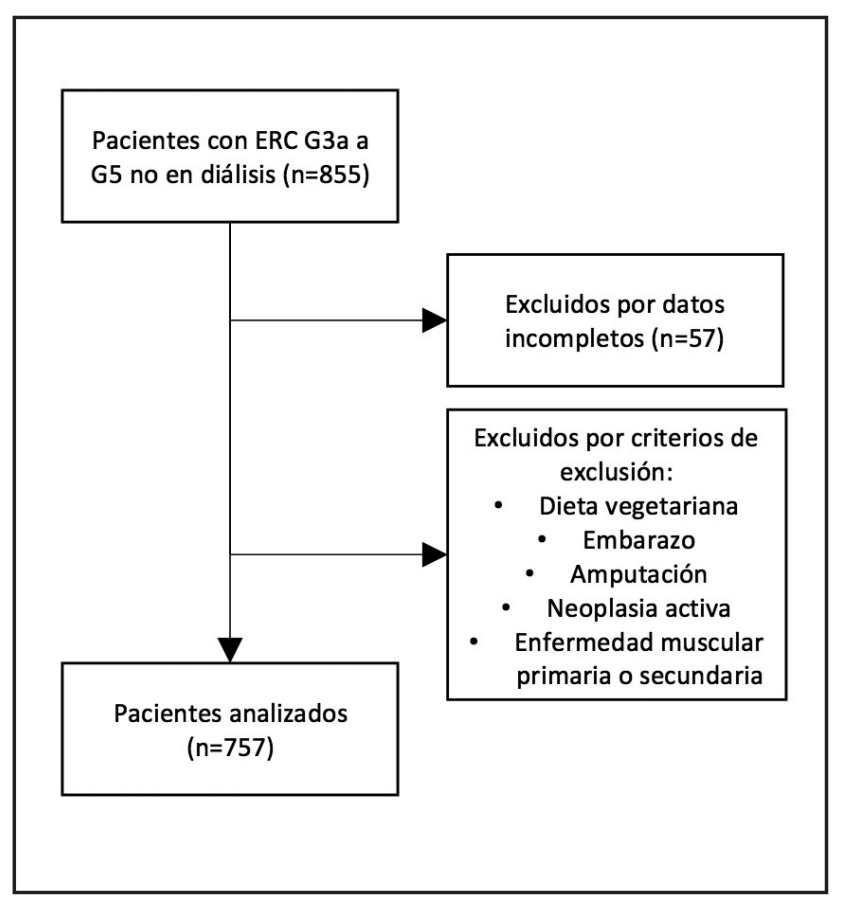

Figura 1. Flujograma de selección de pacientes para el estudio. 
Tabla 1. Características generales de la población.

\begin{tabular}{|c|c|c|c|c|}
\hline Característica & Media $(N=757)$ & Desviación estándar & \multicolumn{2}{|c|}{ IC $95 \%$} \\
\hline Edad (años) & 75.36 & 12.16 & 65.3 & 79.4 \\
\hline Peso $(\mathrm{Kg})$ & 67.3 & 12.42 & 66.7 & 77.8 \\
\hline Talla $(\mathrm{cm})$ & 163.53 & 8.2 & 160.5 & 170.2 \\
\hline $\operatorname{IMC}\left(\mathrm{Kg} / \mathrm{m}^{2}\right)$ & 25.11 & 4.14 & 24.3 & 28.5 \\
\hline Creatinina & 1.86 & 0.97 & 1.35 & 3.1 \\
\hline TFG por $\mathrm{CG}(\mathrm{mL} / \mathrm{min})$ & 35.08 & 12.59 & 27.69 & 42.97 \\
\hline TFG por CG corregido por $\mathrm{SC}\left(\mathrm{mL} / \mathrm{min} / 1.73 \mathrm{~m}^{2}\right)$ & 34.52 & 11.34 & 26.66 & 41.17 \\
\hline TFG por MDRD4 $\left(\mathrm{mL} / \mathrm{min} / 1.73 \mathrm{~m}^{2}\right)$ & 39.81 & 13.20 & 26.99 & 47.34 \\
\hline TFG por CKD-EPI $\left(\mathrm{mL} / \mathrm{min} / 1.73 \mathrm{~m}^{2}\right)$ & 37.31 & 12.71 & 25.72 & 45.11 \\
\hline Albúmina sérica (g/dL) & 4.03 & 0.52 & 3.29 & 4.39 \\
\hline Depuración de creatinina (mL/min) & 44.57 & 19.85 & 36.14 & 63.52 \\
\hline
\end{tabular}

características de los individuos incluidos se muestran en la Tabla 1.

En $37.12 \%$ de los casos se encontró sobrepeso y obesidad en $11.2 \%$. La frecuencia de los estadios de ERC fue diferente para cada una de las fórmulas utilizadas (Figura 2). La fórmula de CG-SC clasificó un porcentaje mayor de casos en ERC G3b comparado con la fórmula de MDRD-4 y CKD-EPI. A su vez, con CG y CG-SC se evidenció una mayor proporción de pacientes en ERC G4, 32.23 y 30.65\%, respectivamente.

La medición de albúmina sérica fue realizada a 508 pacientes (media de $4.0 \pm 0.5 \mathrm{~g} / \mathrm{dL}$ ), el $90.55 \%$ con niveles normales y $9.48 \%$ restante con hipoalbuminemia. La albuminuria promedio fue de $481.58 \pm 305.73 \mathrm{mg} / \mathrm{gr}$ (rango de $1-5009 \mathrm{mg} / \mathrm{gr})$; se obtuvo en un $53.77 \%$ de las mediciones una albuminuria en rango de A1 (<30 mg/gr), 32.43\% en rango de A2 (30-300 mg/gr) y $13.8 \%$ albuminuria en rango de A3 (>300 mg/gr).

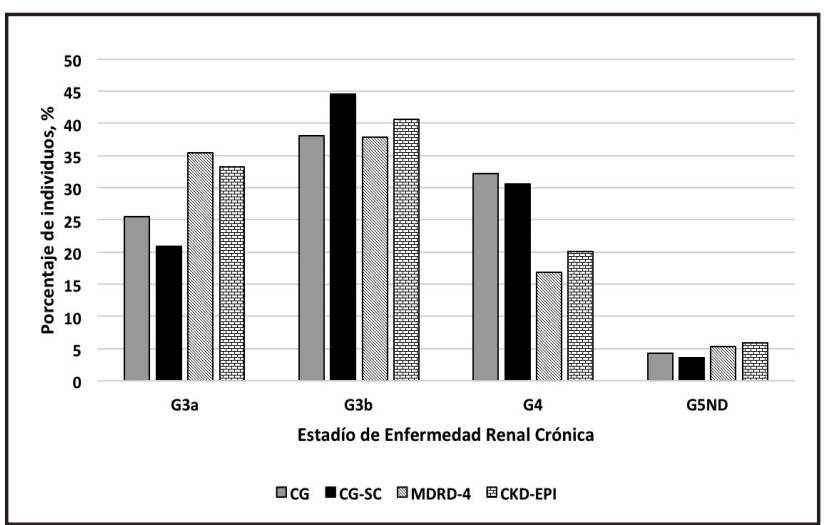

Figura 2. Estadios de la Enfermedad Renal Crónica según las diferentes fórmulas para el cálculo de la tasa de filtración glomerular.

CG: Cockroft-Gault; CG-SC: Cockroft-Gault corregido por la superficie corporal; MDRD-4: modificación de la dieta en la enfermedad renal de cuatro variables; CKD-EPI: fórmula de epidemiología de la enfermedad renal crónica; ND: no en diálisis.
El desempeño de las diferentes fórmulas para estimar la TFG se muestra en la Tabla 2. Todas las fórmulas tuvieron un pequeño sesgo con relación a la FG por CKD-EPI. El sesgo fue negativo para la FGeCG y la FGeCG-SC (-2.23 [-2.82 a -1.65$]$ y $-2.8[-3.24$ a -2.37$] \mathrm{mL} / \mathrm{min} / 1.73 \mathrm{~m}^{2}$ respectivamente) y positivo para la FGeM (2.49 [2.32 a 2.65] $\left.\mathrm{mL} / \mathrm{min} / 1.73 \mathrm{~m}^{2}\right)$. La precisión fue similar entre la FGeCG y la FGeCG-SC (-18.2 a 13.7 y -14.7 a $9.1 \mathrm{~mL} / \mathrm{min} / 1.73 \mathrm{~m}^{2}$ respectivamente); la más precisa fue la FGeM (-2.1 a 7.1 $\mathrm{mL} / \mathrm{min} / 1.73 \mathrm{~m}^{2}$ ). La fórmula más exacta fue la FGeM con una proporción de mediciones dentro del 15 y $30 \%$ en 97.1 y $99.74 \%$ de las mediciones respectivamente, y la menos exacta fue la FGeCG con 59.7 y $81.77 \%$ de las mediciones dentro de 15 y $30 \%$ respectivamente. El coeficiente de correlación de concordancia entre la FGeSO y la FGeM fue de 0.97, con la FGeCG de 0.78 y con la FGeCG-SC de 0.85 .

Las Figuras 3-5 muestran los gráficos de dispersión para cada ecuación y las diferencias entre la FGeM, FGeCG, FGeCG-CS y la FGeSO. La gráfica de Bland-Altman de la FGeCG tiene un límite de concordancia amplio con predominio de subestimación de las medidas, pero con un

Tabla 2. Desempeño de las fórmulas para el cálculo de tasa de filtración glomerular en comparación con CKD-EPI.

\begin{tabular}{|c|c|c|c|c|}
\hline & \multirow{2}{*}{$\begin{array}{c}\text { Sesgo } \\
*\end{array}$} & \multicolumn{2}{|c|}{ Precisión } & \multirow{2}{*}{$\begin{array}{c}\text { Exactitud } \\
\text { P30 (\%) }\end{array}$} \\
\hline & & LI & LS & \\
\hline FGeCG-FGeSO & -2.23 & -18.21 & 13.74 & 81.77 \\
\hline FGeCG-SC-FGeSO & -2.8 & -14.75 & 9.14 & 90.80 \\
\hline FGeM-FGeSO & 2.49 & -2.12 & 7.09 & 99.74 \\
\hline \multicolumn{5}{|c|}{$\begin{array}{l}\text { FGeCG: filtración glomerular por la fórmula de Cockroft-Gault; } \\
\text { FGeCG-SC: filtración glomerular por la fórmula de Cockroft-Gault corregida para } \\
\text { la superficie corporal; } \\
\text { FGeM: filtración glomerular por la fórmula de MDRD-4; } \\
\left.\text { *(mL/min/l.73 } \mathrm{m}^{2}\right) ; \\
\text { LI: límite inferior }\left(\mathrm{mL} / \mathrm{min} / 1.73 \mathrm{~m}^{2}\right) ; \\
\text { LS: límite superior }\left(\mathrm{mL} / \mathrm{min} / 1.73 \mathrm{~m}^{2}\right)\end{array}$} \\
\hline
\end{tabular}


predominio de mediciones de sobrestimación en ERC G4 y G5, lo cual es más evidente en la gráfica de la FGeCG-SC. Por el contrario, la gráfica de Bland-Altman de la FGeM

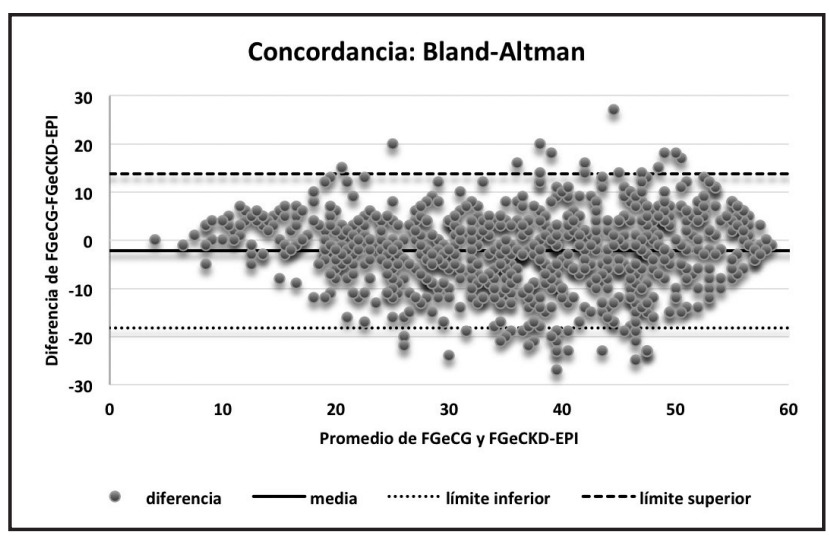

Figura 3. Concordancia Bland-Altman entre FGeCG y FGeCKD-EPI.

CG: Cockroft-Gault; CKD-EPI: fórmula de epidemiología de la enfermedad renal crónica; FGeCG: filtración glomerular estimada por CG; FGeCKD-EPI: filtración glomerular por $C K D-E P I$

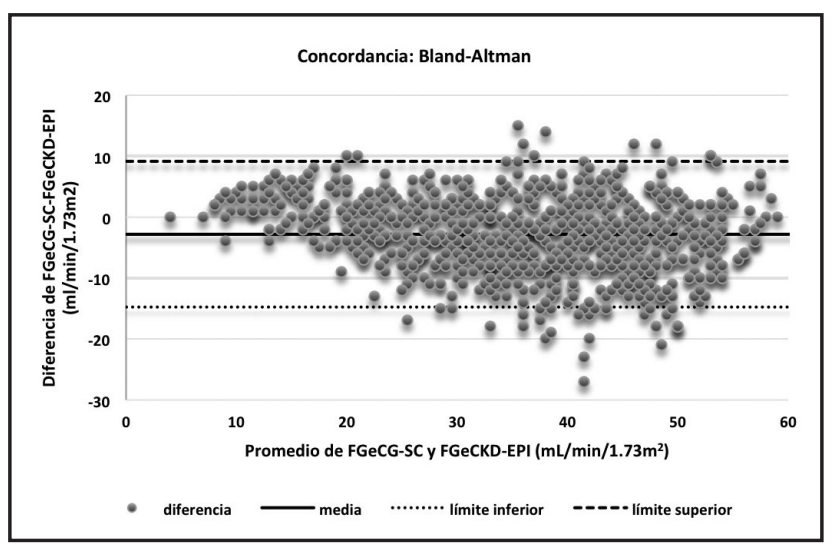

Figura 4. Concordancia Bland-Altman entre FGeCG-SC y FGeCKD-EPI.

CG-SC: Cockroft-Gault corregida por la superficie corporal; CKD-EPI: fórmula de epidemiología de la enfermedad renal crónica; $F G e C G$-SC: filtración glomerular estimada por CG-SC; FGeCKD-EPI: filtración glomerular estimada por CKD-EPI.

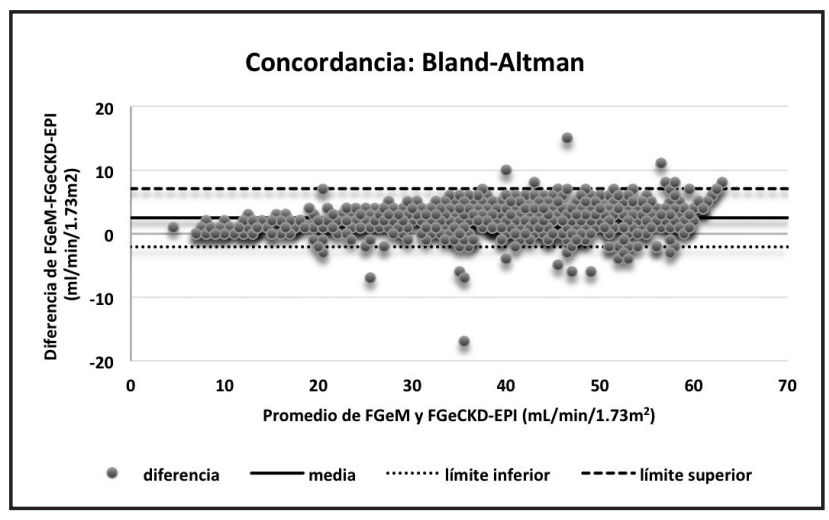

Figura 5. Concordancia Bland-Altman entre FGeM y FGeCKD-EPI

MDRD4: modificación de la dieta en la enfermedad renal de cuatro variables; CKD-EPI: fórmula de epidemiología de la enfermedad renal crónica; FGeM: filtración glomerular estimada por MDRD-4; FGeCKD-EPI: filtración glomerular estimada CKD-EPI. tiene un límite de concordancia estrecho con predominio de sobreestimación de las medidas, pero con mediciones alrededor de cero (0) en ERC G4 y G5.

\section{Discusión}

Todas las fórmulas de estimación de la TFG deben validarse en la población donde se van a utilizar. El presente estudio es el primero que intenta validar en población colombiana el sesgo, la precisión, exactitud y concordancia entre cada una de las fórmulas para la medición de la TFG usando como estándar de oro la TFG estimada por la fórmula CKD-EPI.

En nuestro estudio encontramos que la fórmula de MDRD-4 tuvo el mejor rendimiento en cuanto a precisión, exactitud y concordancia con relación a la fórmula CKDEPI. La precisión no superó el límite establecido de un rango de límites de concordancia de $10($ de -5 a +5$)$, siendo mejor la precisión en el límite de subestimación de la FGeSO. La exactitud es casi perfecta y la concordancia en rango de sustancial. Por su parte, la estimación de la TFG a partir de las ecuaciones de CG y CG-SC mostró gran dispersión respecto a FGeSO, presentando ambas un rango de límites de concordancia por encima del establecido, con una exactitud limítrofe sólo para la FGeCG-SC, pero ambas con una concordancia pobre.

En Colombia no se ha validado la fórmula CKD-EPI, pero ha sido validada internacionalmente, incluyendo población latinoamericana. En 2014 un estudio realizado en mexicanos sanos de edad promedio de 35 años, tomó como referencia valores de medición directa de la TFG obtenidos por medio de un isótopo o radiofármaco (Tc-DTPA: Tc99m-DTPA [ácido dietilentriaminopentacético]) y posteriormente, se comparó con los valores de la TFG estimados con CDKEPI y MDRD-4. Se encontró una mayor concordancia de la TFG calculada por CKD-EPI con los valores de referencia de medición directa (23). Resultados similares obtuvieron Veronese FV, et al (24) en un estudio que incluía pacientes sanos, diabéticos y con ERC. A pesar de tener características poblacionales diferentes, los dos estudios demostraron que CKD-EPI predice mejor la TFG. Sin embargo, en Colombia, la CAC categoriza la ERC por la FGeCG (14). No obstante la FGeCG no permite generalizar sus resultados teniendo en cuenta la presencia de gran heterogeneidad a medida que se incrementa el IMC $(25,26)$ y siendo su rendimiento inferior a la TFG estimada por la fórmula MDRD-4 y CKD-EPI como se ha reportado en los estudios anteriores, y otros en diversos grupos poblacionales $(27,28)$. Estos hallazgos son consistentes con nuestros resultados.

Kuo CF y cols, en un estudio realizado en 32542 individuos sanos sometidos a estudios de rutina compararon la TFG estimada por MDRD de seis variables (MDRD-6), MDRD corregida para población china y CG. La estimación de la TFG, la asignación de factores de riesgo para ERC y su estratificación difirió significativamente entre las tres ecuaciones, especialmente en el caso de la ecuación de CG. La fórmula de CG produjo una TFG más baja y 
paradójicamente mediciones más altas en pacientes con hiperuricemia, hipertensión, síndrome metabólico que en aquellos sin esos factores de riesgo. En este estudio se desaconseja, al igual que en el nuestro, el uso de la fórmula de CG para el tamizaje de la ERC y se sugiere la fórmula MDRD corregida para población china en esa población (29).

Por otro lado, los datos anteriores contrastan con resultados obtenidos por Liu X y cols quienes en población anciana reportaron la fórmula de CG como la más precisa para la estimación de la TFG en diferentes estadios de enfermedad renal crónica (30). Por esto la importancia de nuestro estudio para validar las fórmulas de CG y MDRD-4 en la población colombiana, tomando como referencia la fórmula CKD-EPI de recomendación mundial y aceptada en nuestro país según las guías adoptadas en 2016. En la población analizada encontramos que el rendimiento de MDRD-4 fue estadísticamente mejor en todas las variables de valoración respecto a la FGeCG y la FGeCG-SC, además, fue la más exacta en la estimación de la TFG. Similar a nuestros resultados, los datos obtenidos por Schwandt A, et al (31) concuerdan en que la fórmula MDRD-4 se comportó mejor globalmente, tuvo una mejor precisión y menor sesgo. En esta última investigación, se utilizó como parámetro estándar de oro la TFG estimada por la depuración de creatinina en orina de 24 horas.

Chudek J, et al (32) también compararon el rendimiento de las ecuaciones de MDRD-4, MDRD-6 y CG específicamente en pacientes de 65 años o mayores. Se encontró la mejor concordancia, precisión y exactitud con MDRD-6 en comparación con CG para todos los grupos de ERC. Resultados afines a favor de la precisión de MDRD en pacientes añosos se lograron evidenciar en otro estudio conducido por Aras S y cols (33).

Por último, Zaman SB (34) en un estudio que incluyó 4042 pacientes diabéticos tipo 2, reportó un coeficiente de correlación entre la TFG estimada por CKD-EPI y MDRD4 de 0.90 IC $95 \%$ (0.83 - 0.97, p < 0.001). Sin embargo, este estudio encontró que el coeficiente de correlación entre la ecuación CKD-EPI y CG fue 0.56 (IC 95\%: 0.44 - $0.67, p<0.0001$ ), incluso más bajo que el encontrado en nuestra casuística.

El presente estudio tiene como fortaleza el haberse realizado en un número significativo de pacientes con ERC no en diálisis y comparar por primera vez la FGeSO (CKD-EPI), de recomendación a nivel mundial y adoptada en Colombia en las guías de ERC 2016, con las fórmulas de estimación alternas usadas en Colombia, la MDRD-4 y la utilizada por la CAC, la FGeCG-SC. Pese a que los resultados son sólidos y de significancia clínica por reportarse un análisis global de los datos de pacientes de la ciudad de Cartagena, tiene como debilidades no tener en cuenta un análisis estratificado por estadio de la ERC, subgrupos de pacientes de acuerdo con grupos etarios, pesos promedios y extremos que puedan demostrar hallazgos similares.

\section{Conclusión}

Nuestros hallazgos sugieren que la clasificación en Colombia de las categorías de ERC debería basarse en la fórmula CKD-EPI como se recomienda a nivel internacional por validaciones realizadas con mediciones directas con inulina y radiofármacos. Utilizar como fórmula alterna la de CG-SC no es recomendable a pesar de su facilidad para uso manual, por demostrarse en este estudio es de baja precisión y exactitud, lo cual puede generar en esta población añosa una mayor clasificación de pacientes en etapas tempranas como la ERC G3a, con las implicaciones que tiene para el paciente y el sistema de salud.

En consecuencia, la fórmula alterna más precisa, exacta y concordante para estimar la filtración glomerular en esta población colombiana es la MDRD-4 aun cuando tendría la misma necesidad que la CKD-EPI de utilizar medios en línea para su cuantificación. La fórmula de Cockroft-Gault tiene una baja precisión, exactitud limítrofe y una pobre concordancia con la fórmula de CKD-EPI. Se sugiere no utilizar la estimación de la TFG con la fórmula de CG-SC para clasificar la ERC en Colombia.

\section{Referencias}

1. Coresh J, Stevens LA. Kidney function estimating equations : where do we stand?. Curr Opin Nephrol Hypertens. 2006 May; 15(3): 276-84.

2. Alaini A, Malhotra D, Rondon-Berrios H, Argyropoulos CP, Khitan ZJ, Raj DSC, et al. Establishing the presence or absence of chronic kidney disease: Uses and limitations of formulas estimating the glomerular filtration rate. World J Methodol. 2017 Sep 26; 7(3): 73-92.

3. Van Rijn MHC, Metzger M, Flamant M, Houillier P, Haymann J-P, Van den Brand JAJG, et al. Performance of creatinine-based equations for estimating glomerular filtration rate changes over time. Nephrol Dial Transplant. 2018;(September):1-9.

4. Higdon EA, Kimmons LA, Jr BTD, Hudson JQ. Disagreement in Estimates of Kidney Function for Drug Dosing in Obese Inpatients. J Pharm Pract. 2019 Feb; 32(1): 41-47.

5. Pérez Cabeza AI, Chinchurreta Capote PA, González Correa JA, Ruiz Mateas F, Rosas Cervantes G, Rivas Ruiz F, et al. Discrepancies between the use of MDRD-4 IDMS and CKD-EPI equations, instead of the Cockcroft-Gault equation, in the determination of the dosage of direct oral anticoagulants in patients with non-valvular atrial fibrillation. Med Clin (Barc). 2018 Feb 9; 150(3): 85-91.

6. Miller WG, Jones GRD. Estimated Glomerular Filtration Rate; Laboratory Implementation and Current Global Status. Adv Chronic Kidney Dis. 2018 Jan; 25(1): 7-13

7. Hofman M, Binns D, Johnston V,Siva S, Thompson M, Eu P, et al. 68Ga-EDTA $\mathrm{PET} / \mathrm{CT}$ imaging and plasma clearance for glomerular filtration rate quantification: comparison to conventional 51Cr-EDTA. J Nucl Med. 2015 Mar; 56(3): 405-9.

8. Hall PM, Rolin H. Iothalamate clearance and its use in large-scale clinical trials. Curr Opin Nephrol Hypertens. 1995 Nov; 4(6): 510-3.

9. Yamaguchi T, Higashihara E, Okegawa T, Miyazaki I, Nutahara K. Optimal equation for estimation of glomerular filtration rate in autosomal dominant polycystic kidney disease: influence of tolvaptan. Clin Exp Nephrol. 2018; 22(5): 1213-23.

10. Cristelli MP, Cofán F, Rico N, Trullàs JC, Manzardo C, Agüero F, et al. Estimation of renal function by CKD-EPI versus MDRD in a cohort of HIV-infected patients : a cross-sectional analysis. 2017; $1-7$.

11. Dou Y, Sun X, Liu D, Zhang L, Xiao J, Cheng G, et al. Accuracy of glomerular filtration rate equations for chronic kidney disease patients at the G3a stage : a single - center cross - sectional study. BMC Res Notes. 2017; 2: 1-5.

12. KDIGO. 2012 Clinical practice guideline for the evaluation and management of chronic kidney disease. Kidney Int 2013; 3: 1-150.

13. Ministerio de protección Social. Guía de Práctica Clínica para el diagnóstico y tratamiento de la Enfermedad Renal Crónica (adopción). [Internet]. Bogotá; 2016 [citado: 15 de diciembre de 2019]. Disponible en: http://gpc.minsalud.gov.co/ gpc_sites/Repositorio/Otros_conv/GPC_e_renal/GPC_enfermedad_renal_adopcion.pdf 
14. Cuenta de alto costo. Situación de la enfermedad renal crónica, la hipertensión arterial y la diabetes mellitus. [Internet] Bogotá; 2017 [citado: 15 de diciembre de 2019]. Disponible en: https://cuentadealtocosto.org/site/images/Publicaciones/2018/Libro_Situacion_ERC_en_Colombia_2017.pdf.

15. Cockcroft DW, Gault MH. Prediction of creatinine clearance from serum creatinine. Nephron. 1976; 16(1): 31-41.

16. Deskur-Smielecka E, Kotlinska-Lemieszek A, Chudek J, Wieczorowska-Tobis K. Assessment of renal function in geriatric palliative care patients - Comparison of creatinine-based estimation equations. Clin Interv Aging. 2017;12:977-83.

17. Du Bois D, Du Bois EF. A formula to estimate the approximate surface area if height and weight be known. 1916. Nutrition. 1989; 5(5): 303-11; discussion 12-3.

18. Levey AS, Coresh J, Greene T, Stevens LA, Zhang YL, Hendriksen S, et al. Using Standardized Serum Creatinine Values in the Modification of Diet in Renal Disease Study Equation for Estimating Glomerular Filtration Rate. 2006; 145(4): $247-54$

19. Levey AS, Stevens LA, Schmid CH, Zhang YL, Castro AF 3rd, Feldman HI, et al. New Equation to Estimate Glomerular Filtration Rate. 2009; 150(9): 604-12.

20. Crawford SB, Kosinski AS, Lin HM, Williamson JM, Barnhart HX. Computer programs for the concordance correlation coefficient. Comput Methods Programs Biomed. 2007;88(1):62-74.

21. Bland JM, Altman DG: Statistical methods for assessing agreement between two methods of clinical measurement. Lancet 1: 307-310, 1986.

22. Stevens LA, Zhang Y, Schmid CH. Evaluating the performance of GFR estimating equations. J Nephrol. 2008;21(6):797-807.

23. Arreola-Guerra JM, Rincón-Pedrero R, Cruz-Rivera C, Belmont-Pérez T, Correa-Rotter R, Niño-Cruz JA. Performance of MDRD-IDMS and CKD-EPI equations in Mexican individuals with normal renal function. Nefrologia. 2014; 34(5): 591-8.

24. Veronese FV, Gomes EC, Chanan J, Carraro MA, Camargo EG, Soares AA, et al. Performance of CKD-EPI equation to estimate glomerular filtration rate as compared to MDRD equation in South Brazilian individuals in each stage of renal function. Clin Chem Lab Med. 2014; 52(12): 1747-54.

25. Eastwood JB, Kerry SM, Plange-rhule J, Micah FB, Antwi S, Boa FG, et al. Assessment of GFR by four methods in adults in Ashanti , Ghana : the need for an eGFR equation for lean African populations. Nephrol Dial Transplant. 2010 Jul; 25(7): 2178-87.

26. Michels WM, Grootendorst DC, Verduijn M, Elliott EG, Dekker FW, Krediet RT. Performance of the Cockcroft-Gault, MDRD, and new CKD-EPI formulas in relation to GFR, age, and body size. Clin J Am Soc Nephrol. 2010 Jun; 5(6): 1003-9.

27. Kamaruzaman L, Mohd R, Zaki FM, Hod R, Aziz AA. Estimating glomerular filtration rate in adult kidney transplant recipients in the Asian population. Saudi J Kidney Dis Transpl. 2019 May-Jun; 30(3): 587-596.

28. Uche CL, Osegbe ID. Comparison of CKD-EPI versus MDRD and CockcroftGault equations to estimate glomerular filtration rate among stable homozygous sickle cell patients in Southwest Nigeria. Clin Chem Lab Med. 2014 Dec; 52(12): 1747-54.

29. Kuo CF, Yu KH, Shen YM, See LC. The Chinese Version of the Modification of Diet in Renal Disease (MDRD) Equation is a Superior Screening Tool for Chronic Kidney Disease among Middle-aged Taiwanese than the Original MDRD and Cockcroft-Gault Equations. Biomed J. 2014 Nov-Dec; 37(6): 398-405.

30. Liu X, Cheng MH, Shi CG, Wang C, Cheng CL, Chen JX, et al. Variability of glomerular filtration rate estimation equations in elderly Chinese patients with chronic kidney disease. Clin Interv Aging. 2012; 7: 409-15.

31. Schwandt A, Denkinger M, Fasching P, Pfeifer M, Wagner C, Zeyfang A, et al. Comparison of MDRD, CKD-EPI, and Cockcroft-Gault equation in relation to measured glomerular filtration rate among a large cohort with diabetes. J Diabetes Complications. 2017 Sep; 31(9): 1376-1383.

32. Chudek J, Kolonko A, Owczarek AJ, Wieczorowska K. Clinical factors increasing discrepancies of renal function assessment with MDRD and Cockcroft - Gault equations in old individuals. Eur Geriatr Med. 2018; 9(5): 713-20.

33. Aras S, Varli M, Uzun B, Atli T, Keven K, Aras S, et al. Comparison of Different Glomerular Filtration Methods in the Elderly: Which Formula Provides Better Estimates? Comparison of Different Glomerular Filtration Methods in the Elderly : Which Formula Provides Better Estimates?. Ren Fail. 2012; 34(4): 435-41.

34.Zaman SB. Detection of Chronic Kidney Disease by Using Different Equations of Glomerular Filtration Rate in Patients with Type 2 Diabetes Mellitus: A CrossSectional Analysis. Cureus. 2017 Jun 14; 9(6): e1352. 